\title{
THE MEASUREMENT OF INTELLECTUAL INFLUENCE
}

\author{
By IGNACIO PALACIOS-HuERTA AND OSCAR VOLIJ ${ }^{1}$
}

\begin{abstract}
This paper examines the problem of measuring intellectual influence based on data on citations between scholarly publications. We follow an axiomatic approach and find that the properties of invariance to reference intensity, weak homogeneity, weak consistency, and invariance to splitting of journals characterize a unique ranking method. This method is different from those regularly used in economics and other social sciences.
\end{abstract}

KEYWORDS: Intellectual influence, citations, ranking methods, consistency.

\section{INTRODUCTION}

ACADEMIC JOURNALS have played an increasingly important role in both the dissemination of new knowledge and the certification of scientific merit throughout the past century. In an attempt to evaluate the quality of scientific publications, several efforts have been made to measure influence. Citations, as a broad form of influence, are often used in these efforts.

The literature on citation analysis is by now vast and growing. It is growing mainly because it enables, at a relatively low cost, a first attempt at rigorously quantifying elusive but important socioeconomic phenomena such as reputation, influence, the quality of scholarly output, the productivity of scholars and academic departments, and others. ${ }^{2}$ Further, citation analysis is used widely as a management tool in hiring, promotion, salary, and other personnel decisions.

The use of citation analysis is so extensive that "counting citations is already a wellestablished method of empirical research in law, economics, sociology, and academic administration" (Posner (2000, p. 382), italics added). However, the use of citation counts must be approached with caution, as the number of citations may be a poor proxy of what is really of interest. Some studies attempt to account for this criticism by weighting citations in certain intuitive but ad hoc ways. For instance, many studies use Garfield's (1972) "impact factor," constructed by the Social Science Citation Index, while others follow Liebowitz and Palmer (1984), who proposed various "impactadjusted" methods for ranking journals.

Given the proliferation of rankings and the important role they often seem to play in personnel decisions and in the study of many socioeconomic phenomena of interest, it is somewhat surprising that neither the authors that propose these ranking methods nor those who use them, have tried to justify them. To the best of our knowledge, Amir (2002) is the only attempt at investigating the properties of ranking methods. $\mathrm{He}$

${ }^{1}$ We thank an editor, Andrew Foster, Yiu Poon, Richard Posner, Michael Rothschild, Roberto Serrano, Giora Slutzki, Stephen Stigler, Jinhua Zhao, and numerous seminar participants for helpful comments. We are grateful to Nir Dagan for leading us to valuable references and to Yaakov Bergman for motivation. We also thank Debora Lewi, Salwa Hammami, and Qiaoying $\mathrm{Wu}$ for their research assistance.

${ }^{2}$ For example, citations are used in Ellison (2002), Laband and Piette (1994a), Tuckman and Leahey (1975), Hamermesh, Johnson, and Weisbrod (1982), Laband and Tollison (2000), Sauer (1988), Posner (2000), and other references therein. 
makes a critical assessment of various impact-adjusted measures, in particular the ones proposed by Liebowitz and Palmer (1984). Given that there is, in principle, a plethora of different ranking methods, there is no obvious reason to prefer one method over another. Adopting a method because it looks reasonable or because it yields introspectively intuitive results is, to say the least, not the best scientific practice. Without investigating the properties of these methods it is simply not possible to establish a reliably meaningful measure of impact or intellectual influence. Posner (2000, p. 383) summarizes these criticisms by indicating that "citation analysis is not an inherently economic methodology; most of it has been conducted without any use of the theories or characteristic techniques of economists."

In this paper we bring economic methodology to bear on the ranking problem. Specifically, we present an axiomatic model for measuring intellectual influence. Thus, the approach we take is different from that in the literature. Rather than assuming arbitrarily a ranking method on intuitive grounds or introspection, we derive a ranking method by requiring a few simple properties. The main result of the analysis is that there is a unique ranking method that satisfies the proposed properties simultaneously. This method, which we call the Invariant method, was first proposed by Pinski and Narin (1976), and is different from the methods regularly used to evaluate scholarly publications in economics and in other social sciences. Interestingly, this method is also at the core of the methodology used by Google to rank web sites (see Page et al. (1998)).

Our purpose in this paper is not to claim that any given ranking method based on citations is the correct way of measuring impact, much less quality. Citation analysis, however sophisticated it may be, cannot be a substitute for critical reading and expert judgment. However, to the extent that the data on citations contain valuable information that can be used to address several empirical questions of interest, we should ask why a method is reasonable and informative. We thus approach this question by axiomatically characterizing a ranking method, and by comparing different methods according to the properties they satisfy and those they fail to satisfy.

\section{THE INTELLECTUAL INFLUENCE OF A PUBLICATION}

\subsection{The Model}

Let $\mathcal{J}$ be a nonempty set of journals. This set is to be interpreted as the universe of all potential journals. Let $J \subseteq \mathcal{J}$ be a finite subset of journals. A citation matrix for $J$ is a $|J| \times|J|$ nonnegative matrix $\left(c_{i j}\right)$. For each $i, j \in J, c_{i j}$ represents the number of citations to journal $i$ by journal $j$, or the number of references of journal $j$ to journal $i$. All vectors are column vectors. For a vector $v,\|v\|$ denotes the 1 -norm of $v$, namely $\|v\|=$ $\sum_{i \in J}\left|v_{i}\right|$. The diagonal matrix with $d_{1}, \ldots, d_{n}$ as its main diagonal entries is denoted by $\operatorname{diag}\left(d_{1}, \ldots, d_{n}\right)$. Given a matrix of citations $C=\left(c_{i j}\right)$, we let $c_{j}=\sum_{i \in J} c_{i j}$ be the sum of $j$ 's references and define $D_{C}=\operatorname{diag}\left(c_{j}\right)_{j \in J}$ to be the diagonal matrix with the sums of the journals' references as its main diagonal. Further, the matrix $C D_{C}^{-1}$ will be called the normalized matrix of $C$ and it is readily seen to be a stochastic matrix (the entries of each of its columns add up to one).

Given a matrix of citations $C$ for $J$, we say that journal $i$ is cited by journal $j$ if $c_{i j}>0$. We say that journal $i$ impacts journal $j$ if there is a finite sequence $i_{0}, \ldots, i_{n}$, with $i_{0}=i$ and $i_{n}=j$, such that for all $t=1, \ldots, n$, journal $i_{t-1}$ is cited by journal $i_{t}$. Journals 
$i$ and $j$ communicate if either $i=j$ or if they impact each other. It is easy to see that the communication relation is an equivalence relation and, therefore, it partitions the set $J$ of journals into equivalence classes, which we call communication classes. A discipline is a communication class $J^{\prime} \subseteq J$ such that no journal in $J \backslash J^{\prime}$ impacts any journal inside $J^{\prime}$. Since we are interested in rankings within a single discipline, we will restrict attention to citation matrices whose set of journals constitutes a single discipline. ${ }^{3}$ This leads to the following definition:

DEFINITION 1: A ranking problem is a triple $\langle J, a, C\rangle$, where $J \subseteq \mathcal{J}$ is a set of journals, $a=\left(a_{i}\right)_{i \in J}$ is the vector of the number of articles they published, and $C=$ $\left(c_{i j}\right)_{(i, j) \in J \times J}$ is a citation matrix for $J$ with $J$ as its only discipline.

The primitives of a ranking problem consist of the relevant set of journals, the number of articles published in each journal in the relevant period, and the corresponding matrix of citations. Clearly, the choice of the set of journals is very important and will generally affect the results of the implementation of a ranking method. In our analysis, however, we take as given the set of journals, and deal with the problem of measuring influence within this set. The entries in the citation matrix represent the number of references made by the journals during a citing period to the same journals during a given cited period.

There are two classes of problems that will play an important role in our analysis. One class consists of those problems in which every journal has the same number of articles, i.e., $a_{i}=a_{j}$ for all $i, j \in J$. These problems will be called isoarticle problems. The second class consists of those problems such that every journal has the same average number of references; i.e., $c_{i} / a_{i}=c_{j} / a_{j}$ for all $i, j \in J$. The value $c_{j} / a_{j}$ will be called $j$ 's reference intensity, and problems where all journals have the same reference intensity will be called homogeneous.

We are interested in building a cardinal ranking of the journals in $J$, namely a nonzero vector of nonnegative valuations $\left(v_{j}\right)_{j \in J}$. Each $v_{j}$ is to be interpreted as the value of a representative article in journal $j$. Since only relative values matter, we can normalize the vector of valuations so that they add up to 1 . Denote the set of all possible vectors of valuations of $J$ by $\Delta_{J}$. That is, $\Delta_{J}=\left\{\left(v_{j}\right)_{j \in J}: v_{j} \geq 0, \sum_{j \in J} v_{j}=1\right\}$. Further, $\Delta=\bigcup_{J \subseteq \mathcal{J}} \Delta_{J}$.

DEFINITION 2: Let $\mathcal{R}$ be the set of all ranking problems. A ranking method is a function $\phi: \mathcal{R} \rightarrow \Delta$, that assigns to each ranking problem $\langle J, a, C\rangle$ a vector of valuations $v \in \Delta_{J}$.

Given a vector $\left(a_{i}\right)_{i \in J}$ of number of articles, we will denote by $A$ the diagonal matrix $\operatorname{diag}\left(a_{i}\right)_{i \in J}$. The following are some examples of different ranking methods:

1. The Counting method awards each journal a value that is proportional to the average number of citations of its articles. Formally: $\phi_{C}: \mathcal{R} \rightarrow \Delta$ is defined by

$$
\phi_{C}(J, a, C)=\left(\frac{\sum_{j \in J} c_{i j} / a_{i}}{\sum_{k \in J} \sum_{j \in J} c_{k j} / a_{k}}\right)_{i \in J} .
$$

${ }^{3}$ Such matrices are known as nonnegative, irreducible matrices. 
2. The Modified Counting method awards each journal a value that is proportional to the average number of non-self-citations of its articles. Formally: $\phi_{M C}: \mathcal{R} \rightarrow \Delta$ is defined by

$$
\phi_{M C}(J, a, C)=\left(\frac{\sum_{j \in J \backslash\{i\}} c_{i j} / a_{i}}{\sum_{k \in J} \sum_{j \in J \backslash\{k\}} c_{k j} / a_{k}}\right)_{i \in J} .
$$

3. The Liebowitz-Palmer method $\phi_{L P}: \mathcal{R} \rightarrow \Delta$ assigns to each ranking problem $R=$ $\langle J, a, C\rangle$ the unique vector $\left(v_{i}\right)_{i \in J} \in \Delta_{J}$ that solves

$$
v_{i}=\frac{\sum_{j \in J} \frac{c_{i j}}{a_{i}} v_{j}}{\sum_{k \in J} \sum_{j \in J} \frac{c_{k j}}{a_{k}} v_{j}}, \quad i \in J,
$$

or in matrix notation

$$
v=\frac{A^{-1} C v}{\left\|A^{-1} C v\right\|} .
$$

4. The Invariant method $\phi_{I}$ assigns to each ranking problem $R=\langle J, a, C\rangle$, the unique member of $v \in \Delta_{J}$ that satisfies

$$
v_{i}=\sum_{j \in J} \frac{c_{i j}}{a_{i}} \frac{a_{j}}{c_{j}} v_{j}, \quad i \in J,
$$

or in matrix notation $A^{-1} C D_{C}^{-1} A v=v$.

The Counting and Modified Counting methods were first used in Bush, Hamelman, and Staaf (1974). The Liebowitz-Palmer method was proposed by Liebowitz and Palmer (1984), and it is the predominant method used to rank economics journals. The Invariant method was first proposed by Pinski and Narin (1976), and it is at the core of the PageRank method used by Google to rank web pages (see, e.g., Page et al. (1998)).

That the Invariant method is well-defined follows from the fact that the normalized matrix $C D_{C}^{-1}$ is an irreducible stochastic matrix and that every irreducible stochastic matrix has a unique invariant distribution. The fact that the Liebowitz-Palmer method is well defined is a corollary of the Perron-Frobenius theorem for irreducible matrices. ${ }^{4}$

Note that both the Liebowitz-Palmer and the Invariant methods assign to journal $i$ a value that is a weighted average of some function of its citations. According to these measures, not all citations have the same value. Citations by important journals are more valuable than citations by less important journals. But the importance of a journal is determined endogenously and simultaneously with the importance of all other journals.

Both these methods yield a positive eigenvector of an appropriately adjusted matrix of citations. The Liebowitz-Palmer method calculates the positive eigenvector of the

${ }^{4}$ Specifically, note that $\phi_{L P}(J, a, C)$ is a characteristic vector in $\Delta_{J}$ of the nonnegative and irreducible matrix $A^{-1} C$ and recall that every irreducible, nonnegative, $|J| \times|J|$ matrix $M$ has exactly one eigenvector in $\Delta_{J}$ (see Minc (1988, Theorem 4.4)). 
matrix $A^{-1} C$, while the Invariant method calculates the positive eigenvector of the matrix $A^{-1} C D_{C}^{-1} A$. The entry $c_{i j} / a_{i}$ of the matrix $A^{-1} C$ is the average number of citations that an article in journal $i$ gets from journal $j$. This is the underlying measure of direct impact (of a typical article in $i$ on a typical article in $j$ ) that the Liebowitz-Palmer method takes into account. The Invariant method, on the other hand, controls for reference intensity by dividing the value $c_{i j} / a_{i}$ by $c_{j} / a_{j}$, that is, by the reference intensity of the articles in $j$. Therefore, the measure of direct impact of journal $i$ on journal $j$ that underlies the Invariant method is the average number of citations of an article in $i$ out of the average number of references by a typical article of $j$.

We next introduce some basic properties that ranking methods may satisfy. In order to motivate the first property, consider a ranking problem $\langle J, a, C\rangle$. For each $j \in J$, journal $j$ 's list of references, $\left(c_{i j}\right)_{i \in J}$, represents journal $j$ 's opinions about the journals in $J$. These opinions are given by the ratios $c_{i j} / c_{j}$ of $j$ 's references to the different journals. They would not change if journal $j$ were to modify the number of references by multiplying them by a constant $\lambda_{j}>0$, thus turning the vector $\left(c_{i j}\right)_{i \in J}$ into the vector $\left(\lambda_{j} c_{i j}\right)_{i \in J}$. The first property requires that the ranking method not be affected by such changes. In other words, all else equal, the length of the reference section should not matter.

PROPERTY 1: A ranking method $\phi$ satisfies invariance with respect to reference intensity if for every ranking problem $\langle J, a, C\rangle$ and for every diagonal matrix $\Lambda=\operatorname{diag}\left(\lambda_{j}\right)_{j \in J}$ with strictly positive diagonal entries, $\phi(J, a, C \Lambda)=\phi(J, a, C)$.

The idea behind this property is that each journal should have one vote. If journal $j$ refers to many articles in different journals, then that journal's vote is divided among the cited journals.

The next two properties concern homogeneous problems, namely those where all journals have the same reference intensity. The first one is motivated by the analysis in Stigler, Stigler, and Friedland (1995).

PROPERTY 2: The ranking method $\phi$ satisfies weak homogeneity if for all homogeneous and isoarticle two-journal problems $R=\langle\{i, j\}, a, C\rangle, \phi_{i}(R) / \phi_{j}(R)=c_{i j} / c_{j i}$. We say that $\phi$ satisfies homogeneity if the above condition holds for all homogeneous two-journal problems, not necessarily isoarticle ones.

This property says that in two-journal problems where both journals have the same number of articles and the same reference intensity, the relative valuation of a journal should be the ratio of their mutual citations. The value $c_{i j}$ is a measure of $i$ 's direct influence on $j$. Thus, the ratio $c_{i j} / c_{j i}$ represents the direct influence of journal $i$ on journal $j$ relative to the direct influence of journal $j$ on journal $i$. The importance of these ratios was stressed in Stigler, Stigler, and Friedland (1995) who calculated them for a group of nine core journals. In building a desirable ranking method, though, one would like to take into account not only the direct influence of the journals on each other, but also their indirect influence. Thus, though conveying important information, these ratios are not, per se, a perfect index of the journals' total impact. In a two-journal problem, however, the value $c_{i j}$ is a measure of the total impact of journal $i$ on journal $j$. Stigler, Stigler, and Friedland (1995, p. 336) admit that "these sender-receiver ratios are influenced by the varying number of citations in articles published by each journal," but 
they are not clear as to whether that variation calls for correction. In any case, our weak homogeneity property requires that the ratio of valuations be proportional to the ratio of mutual citations only in two-journal isoarticle, homogeneous problems. In this way, the varying number of citations and the effect of indirect influences across journals are not an issue.

The third property will allow us to relate large problems to smaller ones. The idea is to extend a ranking method of few journals to a ranking method of more journals in a consistent way. To formalize what we mean by consistency, we first need some definitions.

Let $R=\left\langle J,\left(a_{i}\right)_{i \in J},\left(c_{i j}\right)_{(i, j) \in J \times J}\right\rangle$ be a ranking problem, and let $k \in J$. The reduced ranking problem with respect to $k$ is $R^{k}=\left\langle J \backslash\{k\},\left(a_{i}\right)_{i \in J \backslash\{k\}},\left(c_{i j}^{k}\right)_{(i, j) \in J \backslash\{k\} \times J \backslash\{k\}}\right\rangle$, where:

$$
c_{i j}^{k}=c_{i j}+c_{k j} \frac{c_{i k}}{\sum_{t \in J \backslash k\}} c_{t k}} \quad \text { for all } i, j \in J \backslash\{k\} .
$$

Note that since $\left(c_{i j}\right)_{(i, j) \in J \times J}$ is irreducible, $\sum_{t \in J \backslash\{k\}} c_{t k}>0$, and hence, $R^{k}$ is well-defined. Further, $\left(c_{i j}^{k}\right)_{(i, j) \in J \backslash\{k\} \times J \backslash\langle k\}}$ is itself irreducible.

The reduced problem represents the following situation. Suppose we want to rank the journals in $J$ and our computer cannot deal with $|J| \times|J|$ matrices but only with $(|J|-1) \times(|J|-1)$ matrices. Therefore, we need to resize our problem and abstract from one journal in our data set, say journal $k$. Still, we are interested in the relative values of all the remaining journals. If we eliminated journal $k$ from the matrix, namely if we eliminated its corresponding row and column, we would lose some valuable information. Therefore, we need to "retouch" the matrix so that the information from the missing journal is not lost. In the old matrix, $c_{k j}$ was the number of references to journal $k$ by journal $j$. To keep this information, we may need to redistribute these citations among the other journals. One way to do so is in proportion to the citations by the missing journal $k$. In other words, journal $j$ 's references to journal $k$ could be redistributed back to journals other than $k$ according to journal $k$ 's opinions.

Though not the only way to recover information lost by the need for a smaller matrix, if one accepts it as a reasonable one, we should expect that our ranking method give, at least in homogeneous problems, the same relative valuations to the journals in $J \backslash\{k\}$ both when applied to the original and to the reduced problems. This is the requirement of the next property.

PROPERTY 3: The ranking method $\phi$ satisfies weak consistency if for all homogeneous, isoarticle problems $R=\langle J, a, C\rangle$, with $|J|>2$, and for all $k \in J$,

$$
\frac{\phi_{i}(R)}{\phi_{j}(R)}=\frac{\phi_{i}\left(R^{k}\right)}{\phi_{j}\left(R^{k}\right)} \quad \text { for all } i, j \in J \backslash\{k\} .
$$

We say that $\phi$ satisfies consistency if the above condition holds for all homogeneous problems, not necessarily isoarticle ones.

The property of weak consistency requires from a ranking method that the relative valuations of the journals in a homogeneous problem be unaffected if we apply the method to the reduced problem with respect to $k$. Clearly, the appeal of this property depends on the notion of a reduced problem that one adopts. As we will see, 
weak consistency is satisfied by several ranking methods, including the Invariant and the Liebowitz-Palmer.

The properties introduced so far are sufficient to rank journals with the same number of articles. The next, and last, property will enable us to rank journals with different numbers of articles.

Let $R=\left\langle J,\left(a_{j}\right)_{j \in J},\left(c_{i j}\right)_{(i, j) \in J \times J}\right\rangle$ be a ranking problem. Each journal $j \in J$ will be split into $T_{j} \geq 1$ identical journals (that is, they will have the same profile of references and citations), denoted $\left(j, t_{j}\right)$, for $t_{j}=1, \ldots, T_{j}$. With some abuse of notation, we shall denote by $T_{j}$ both the set and the number of "types" of journal $j$. The resulting ranking problem is $R^{\prime}=\left\langle J^{\prime},\left(a_{\left(j, t_{j}\right)}^{\prime}\right)_{j, t_{j} \in J^{\prime}},\left(c_{\left(i, t_{i}\right)\left(j, t_{j}\right)}^{\prime}\right)_{\left(\left(i, t_{i}\right),\left(j, t_{j}\right)\right) \in J^{\prime} \times J^{\prime}}\right\rangle$, where $J^{\prime}=\left\{\left(j, t_{j}\right): j \in J\right.$, $\left.t_{j} \in T_{j}\right\}, a_{\left(j, t_{j}\right)}^{\prime}=a_{j} / T_{j}$, and $c_{\left(i, t_{i}\right)\left(j, t_{j}\right)}^{\prime}=c_{i j} / T_{i} T_{j}$. We will call the problem $R^{\prime}$ a split of $R$, and we will denote its citation matrix $\left(c_{\left(i, t_{i}\right)\left(j, t_{j}\right)}^{\prime}\right)_{\left(\left(i, t_{i}\right),\left(j, t_{j}\right)\right) \in J^{\prime} \times J^{\prime}}$ by $C^{\prime}$.

We expect a split of a journal not to affect the relative valuations of the articles. This is the requirement imposed by the following property.

PROPERTY 4: A ranking method $\phi$ satisfies invariance to splitting of journals if for all ranking problems $R=\left\langle J,\left(a_{j}\right)_{j \in J},\left(c_{i j}\right)_{(i, j) \in J \times J}\right\rangle$, for all $i, j \in J$, and for all its splittings $R^{\prime}=\left\langle J^{\prime},\left(a_{\left(j, t_{j}\right)}^{\prime}\right)_{j, t_{j} \in J^{\prime}},\left(c_{\left(i, t_{i}\right)\left(j, t_{j}\right)}^{\prime}\right)_{\left(\left(i, t_{i}\right),\left(j, t_{j}\right)\right) \in J^{\prime} \times J^{\prime}}\right\rangle$, we have:

$$
\frac{\phi_{i}(R)}{\phi_{j}(R)}=\frac{\phi_{\left(i, t_{i}\right)}\left(R^{\prime}\right)}{\phi_{\left(j, t_{j}\right)}\left(R^{\prime}\right)} \quad \forall i, j \in J \text { and } \forall t_{i} \in T_{i} \text { and } t_{j} \in T_{j} .
$$

We are now ready to characterize the only ranking method that satisfies all the properties described above.

THEOREM: There is a unique ranking method that satisfies invariance to reference intensity, weak homogeneity, weak consistency, and invariance to splitting of journals. It is the Invariant method $\phi_{I}$.

For the proof see the Appendix.

\subsection{Remarks}

As we also show in the Appendix, the four axioms are independent. In particular, the Liebowitz-Palmer method, which is the one regularly used to rank economics journals, satisfies all the axioms except for invariance to reference intensity. Since this is the main difference between the two methods, we will clarify what this axiom does and does not do.

A ranking method that satisfies invariance is, by definition, homogeneous of degree 0 in each of the opinions of the journals. Thus, such a method is immune to "citation inflation" within journals. However, this method is not immune to citation inflation within the articles themselves when the individual articles' opinions are aggregated into a journal's opinion. Formally, consider journal $j$ with $K$ articles. Article $j_{k}$ 's references to the journals in $J$ are given by $\left(c_{i j_{k}}\right)_{i \in J}$. Letting $c_{* j_{k}}=\sum_{i \in J} c_{i j_{k}}$ denote the sum of article $j_{k}$ 's references, its opinions are given by $\left(c_{i j_{k}} / c_{* j_{k}}\right)_{i \in J}$. Further, since journal $j$ 's vector of references is $\left(c_{i j}\right)_{i \in J}=\left(\sum_{k=1}^{K} c_{i j_{k}}\right)_{i \in J}$, journal $j$ 's opinions are given by

$$
\left(\frac{c_{i j}}{c_{j}}\right)_{i \in J}=\left(\sum_{k=1}^{K} \frac{c_{i j_{k}}}{c_{j}}\right)_{i \in J}=\left(\sum_{k=1}^{K} \frac{c_{i j_{k}}}{c_{* j_{k}}} \frac{c_{* j_{k}}}{c_{j}}\right)_{i \in J} .
$$


It can be seen that journal $j$ 's opinions are a weighted average of the opinions of its articles, where the weight of each article is the proportion of its references out of the total number of the journal's references. Therefore, the greater the number of references an article has, the greater the weight of its opinions in the aggregation of the journal's opinions. This implies that, unless it is a constant function, a method that depends on the journals' opinions will not be immune to citation inflation within the articles. This means, for instance, that if the articles in a particular field make systematically more references than the articles in other fields, then the opinions of the former will have more weight than the opinions of the latter. But this problem is unavoidable as long as one uses as data the matrix $C$ whose columns contain the sum of the references of the articles of the respective journals. In order to provide immunity to citation inflation at the level of the articles, it is necessary to use disaggregated data so that the simple average of each journal's individual articles' opinions, $\left(1 / K \sum_{k=1}^{K}\left(c_{i j_{k}} / c_{* j_{k}}\right)\right)_{i \in J}$, can be calculated. For instance, one could define the data of a ranking problem alternatively as a matrix of citations where each column represents one article published during the citing period and each row represents a journal in the cited period. This disaggregation would allow more information to be taken into account in determining the measure of each journal's influence. We chose to define a ranking problem by means of the aggregated matrix of citations $C$ for simplicity and because this is the way the data are generally used in citation analysis.

Ideally one would like to use disaggregated data of citations from one article to another. However, since older articles cannot cite newer ones, the resulting matrix would not be irreducible. This temporal dimension forces one to aggregate articles into journals (or any other meaningful aggregate), and to consider a journal's citations as coming from papers that are representative of the journals in which they appear. Note, however, that the methodology considered in this paper can be readily applied to ranking web pages, which can link to each other simultaneously. In this atemporal context there is no need to perform any aggregation.

Lastly, one of the key ingredients of a ranking problem as defined in this paper is the number of articles published by each of the journals. The resulting index aims at measuring the influence per article published by these journals. There are some studies, however, that calculate influence measures per page by applying the Liebowitz-Palmer methodology to citations per page instead of citations per article. A first order effect of this approach is that journals with a low pages-per-article ratio will have an increased influence measure relative to high pages-per-article journals. As a second order effect, journals that are highly cited by low pages-per-article journals will also tend to benefit from the per page measure. The same axioms used in this paper, properly interpreted, characterize a unique per-page ranking method that takes as input not the number of articles but the number of pages. We preferred to make the exposition in terms of per article indexes because citations refer to articles, not to pages, and because the evidence does not support a one for one relationship between citations and the length of a paper.

\section{ILLUSTRATIONS FROM ECONOMICS JOURNALS}

This section illustrates the use of the Invariant and the Liebowitz-Palmer (LP) methods for the measurement of influence in economics. These illustrations allow us to evaluate differences in the rankings of journals across methods and differences that arise by simply weighting citations at the level of articles. 
We first report the Invariant rankings and the LP rankings according to their permanuscript impact in a sample of journals. We use data of citations during the year 2000 in these journals to articles published in the same set of journals during the period 1993-1999. The data come from the 2000 Social Sciences Edition of the Journal Citation Reports published by the Institute for Scientific Information. The dataset considers all papers published by these journals, including short papers, comments, and nonrefereed articles. We only adjusted data for the American Economic Review (AER) where we considered separately the papers published in the May issue, which we denoted as AER Papers and Proceedings. The values we obtain, reported in Table I, are rescaled so that for each measure they add up to 1,000 . This table also reports the journals' reference intensities, normalized so that their average is 1.

According to both methods, the first six journals account for roughly half of the total impact. Still, the LP and Invariant methods may in some cases yield substantially different numerical rankings. For instance, according to the Invariant method, Econometrica and the Quarterly Journal of Economics $(Q J E)$ have similar values, and the value of the $A E R$ is about 75 percent the value of Econometrica. According to the LP method, however, the $Q J E$ is the top journal in the sample, while the value of Econometrica, which drops below the value of the $A E R$, is about 72 percent of the value of the QJE. In general, Econometrica and a number of theory journals in economics and econometrics tend to fare better under the Invariant method. The results of the previous section indicate that these differences are due to the fact that the Invariant method satisfies the axiom of invariance with respect to reference intensity, while the LP method does not. Indeed, the reference intensity of journals such as the QJE, AER, and the Journal of Economic Literature (JEL) is much greater than the average reference intensity of the journals in the sample. The data (not reported here) also show that these three journals tend to cite each other, relative to other journals, to a much greater extent. Theory journals, on the other hand, tend to have a lower than average reference intensity and get a small proportion of citations from the $A E R, Q J E$, and $J E L$. Lastly, the $Q J E$ seems to have risen in the rankings and the $A E R$ seems to have dropped since Laband and Piette (1994b) reported their LP estimates.

Influence-weighted citations may also be used as a measure of the importance of individual papers. Table II considers a sample of papers and the citations that these papers received in the year 2000 in the sample of journals considered in the previous table. The three columns show the number of unweighted citations, Invariant-weighted citations, and LP-weighted citations that these papers received. ${ }^{5}$

${ }^{5}$ The average value of an article published in journal $i$ according to the Invariant method is

$$
v_{i}=\sum_{j} \frac{c_{i j} / a_{i}}{c_{j} / a_{j}} v_{j}
$$

Therefore, the value of a particular paper $p_{i}$ published in journal $i$ is given by

$$
v_{p_{i}}=\sum_{j} \frac{c_{p_{i} j}}{c_{j} / a_{j}} v_{j}
$$

Similarly, the value of $p_{i}$ according to the LP method is $v_{p_{i}}=\sum_{j} c_{p_{i} j} v_{j}$. In the table, these values are normalized so that the average number of unweighted citations and weighted citations coincide. The variances of unweighted citations and weighted citations in this sample of highly 
TABLE I

JOURNAL RANKINGS BASED ON PER ARTICLE IMPACT

\begin{tabular}{|c|c|c|c|}
\hline & Invariant Method & LP Method & Reference Intensity \\
\hline Econometrica & 102.6 & 86.9 & 1.1 \\
\hline Quarterly Journal of Economics & 101.4 & 119.6 & 1.5 \\
\hline Journal of Economic Literature & 80.6 & 88.6 & 2.7 \\
\hline American Economic Review & 77.9 & 92.8 & 1.7 \\
\hline Journal of Political Economy & 68.6 & 74.8 & 1.0 \\
\hline Review of Economic Studies & 66.0 & 67.4 & 1.3 \\
\hline Journal of Monetary Economics & 47.3 & 59.9 & 1.8 \\
\hline Journal of Economic Theory & 35.3 & 27.1 & .7 \\
\hline Games and Economic Behavior & 33.4 & 26.0 & 1.1 \\
\hline Journal of Economics Perspectives & 31.8 & 34.6 & .9 \\
\hline Journal of Econometrics & 21.7 & 15.6 & 1.2 \\
\hline Rand Journal of Economics & 20.6 & 16.4 & .8 \\
\hline Economic Theory & 18.7 & 16.9 & 1.0 \\
\hline Journal of Labor Economics & 17.8 & 17.5 & .8 \\
\hline Journal of Human Resources & 17.4 & 16.2 & .8 \\
\hline Journal of Public Economics & 16.7 & 16.8 & 1.0 \\
\hline Review of Economics and Statistics & 16.7 & 17.1 & .9 \\
\hline Econometric Theory & 16.5 & 10.1 & .7 \\
\hline Journal of Risk and Uncertainty & 16.2 & 17.2 & .7 \\
\hline International Economic Review & 16.0 & 15.3 & .9 \\
\hline Journal of Financial Economics & 15.4 & 13.3 & 1.1 \\
\hline Journal of Business and Ec. Statistics & 15.2 & 12.3 & .4 \\
\hline AER Papers and Proceedings & 14.0 & 14.9 & .4 \\
\hline Journal of Applied Econometrics & 13.3 & 12.1 & 1.5 \\
\hline European Economic Review & 13.3 & 14.2 & 1.0 \\
\hline International Journal of Game Theory & 13.2 & 10.6 & .4 \\
\hline Social Choice and Welfare & 12.8 & 7.7 & 1.0 \\
\hline J. of Environmental Ecs. and Management & 12.5 & 9.9 & .7 \\
\hline Economic Journal & 12.2 & 12.6 & .8 \\
\hline Journal of International Economics & 11.7 & 18.2 & 1.5 \\
\hline Journal of Ec. Dynamics and Control & 10.8 & 10.0 & 1.0 \\
\hline Journal of Mathematical Economics & 10.3 & 6.2 & .8 \\
\hline Economic Inquiry & 6.3 & 7.2 & .7 \\
\hline Journal of Ec. Behavior and Organization & 5.3 & 4.9 & .9 \\
\hline Scandinavian Journal of Economics & 4.3 & 4.4 & .5 \\
\hline Economics Letters & 3.2 & 2.8 & .5 \\
\hline Oxford Bulletin of Ecs. and Statistics & 2.7 & 2.2 & 1.0 \\
\hline
\end{tabular}

The results show that weighted rankings can be substantially different from unweighted rankings. In our sample, while there are papers whose relative impact is basically maintained across weighted and unweighted columns, others have drastically

cited papers turn out to be basically identical. However, in a larger sample of 250 randomly selected papers that we also examined, we found that the influence of papers according to weighted measures is much more heterogeneous than according to the unweighted citations. 
TABLE II

WEIGHTED VERSUS UNWEIGHTED CITATIONS FOR INDIVIDUAL ARTICLES ${ }^{\mathrm{a}}$

\begin{tabular}{|c|c|c|c|c|}
\hline ID\# & Article & $\begin{array}{c}\text { Number of } \\
\text { Unweighted } \\
\text { Citations }\end{array}$ & $\begin{array}{l}\text { Invariant- } \\
\text { Weighted } \\
\text { Citations }\end{array}$ & $\begin{array}{c}\text { LP- } \\
\text { Weighted } \\
\text { Citations }\end{array}$ \\
\hline 1 & Rabin (AER, 1993) & 20 & 17.04 & 15.75 \\
\hline 2 & Kandori, Mailath and Rob (Econometrica, 1993) & 18 & 17.07 & 12.31 \\
\hline 3 & Milgrom and Shannon (Econometrica, 1994) & 17 & 18.49 & 13.55 \\
\hline 4 & Galor and Zeira (REStud, 1993) & 14 & 12.54 & 13.78 \\
\hline 5 & Berry, Levinsohn and Pakes (Econometrica, 1995) & 14 & 8.54 & 7.67 \\
\hline 6 & Benabou $(A E R, 1996)$ & 12 & 13.85 & 16.95 \\
\hline 7 & Grossman and Helpman (AER, 1994) & 12 & 7.03 & 5.66 \\
\hline 8 & Persson and Tabellini (AER, 1994) & 11 & 11.92 & 14.07 \\
\hline 9 & Mauro (QJE, 1995) & 11 & 8.14 & 9.42 \\
\hline 10 & Ellison (Econometrica, 1993) & 10 & 12.21 & 9.65 \\
\hline 11 & Jones (JPE, 1995) & 10 & 6.59 & 9.37 \\
\hline 12 & Autor, Katz and Krueger (QJE, 1998) & 9 & 11.83 & 13.90 \\
\hline 13 & Juhn, Murphy and Pierce (JPE, 1993) & 9 & 11.08 & 10.69 \\
\hline 14 & Erev and Roth (AER, 1998) & 9 & 10.69 & 5.87 \\
\hline 15 & Aghion and Bolton (REStud, 1997) & 9 & 9.77 & 10.79 \\
\hline 16 & Easterly and Levine (QJE, 1997) & 9 & 7.24 & 6.54 \\
\hline 17 & Berman, Bound and Griliches (QJE, 1994) & 9 & 6.08 & 5.41 \\
\hline 18 & Piketty (QJE, 1995) & 8 & 10.85 & 12.05 \\
\hline 19 & Manski (REStud, 1993) & 8 & 9.51 & 10.79 \\
\hline 20 & Borjas (AER, 1995) & 8 & 8.83 & 12.12 \\
\hline 21 & Imbens and Angrist (Econometrica, 1994) & 8 & 8.48 & 8.45 \\
\hline 22 & Fehr, Gachter, Kirchsteiger (Econometrica, 1997) & 8 & 5.47 & 5.58 \\
\hline 23 & Galor and Tsiddon (AER, 1997) & 7 & 10.16 & 12.11 \\
\hline 24 & Goldin and Katz (QJE, 1998) & 7 & 9.23 & 9.22 \\
\hline 25 & Epple and Romano (AER, 1998) & 7 & 8.99 & 12.10 \\
\hline 26 & DiNardo, Fortin, Lemieux (Econometrica, 1996) & 7 & 5.81 & 4.34 \\
\hline 27 & Young (REStud, 1998) & 7 & 5.36 & 3.73 \\
\hline 28 & La Porta et al. (JPE, 1998) & 7 & 4.81 & 4.11 \\
\hline 29 & Glaeser, Sacerdote and Scheinkman (QJE, 1996) & 6 & 9.79 & 12.39 \\
\hline 30 & Bolton and Dewatripont (QJE, 1994) & 6 & 8.96 & 8.66 \\
\hline 31 & Townsend (Econometrica, 1994) & 6 & 8.00 & 6.35 \\
\hline 32 & Doms, Dunne and Troske (QJE, 1996) & 6 & 7.83 & 10.15 \\
\hline 33 & Heaton and Lucas (JPE, 1996) & 6 & 2.74 & 1.48 \\
\hline
\end{tabular}

a The column ID\# shows the identification number to which we refer in the text. The column "Article" indicates the last name of the author(s) and in brackets the journal where the paper was published and the publication year. "JPE" refers to the Journal of Political Economy and "REStud" to the Review of Economic Studies.

different relative impacts. For instance, papers \#6 and \#7 both have twelve citations but the former has about two to three times the impact of the latter when citations are weighted. Papers \#23 and \#27, \#29 and \#33, and others provide similarly striking comparisons. In fact, paper \#29's six unweighted citations have a weighted value that is greater than paper \#5's fourteen unweighted citations. These illustrations show that weighting citations can be very important, in particular because papers appear to be quite heterogeneous, even within journals. 
Dept.of Economics, Brown University, Providence, RI 02912,U.S.A.; ipalacios@brown. edu; http:// www.econ.brown.edu/ iph

and

Dept. of Economics, Iowa State University, Ames, IA 50011, U.S.A.; oscar@volij. co.il; http://volij.co.il.

Manuscript received February, 2003; final revision received August, 2003.

\section{APPENDIX A: PROOF OF THE THEOREM}

We first show that the Invariant method satisfies the stated properties. Later we show that no other method satisfies all these properties simultaneously. The Invariant method satisfies invariance to reference intensity since for any citation matrix $C$ and for any diagonal matrix $\Lambda$ with positive diagonal entries, $C$ and $C A$ have the same normalized matrix. To see that it satisfies homogeneity, and a fortiori weak homogeneity, let $R=\langle J, a, C\rangle$ be a two-journal, homogeneous problem. That is, $D_{C}=\kappa A \equiv \kappa \operatorname{diag}\left(a_{1}, a_{2}\right)$ for some $\kappa>0$, and

$$
C=\left(\begin{array}{cc}
\kappa a_{1}-c_{21} & c_{12} \\
c_{21} & \kappa a_{2}-c_{12}
\end{array}\right) \text {. }
$$

But then,

$$
A^{-1} C D_{C}^{-1} A\left(c_{12}, c_{21}\right)^{T}=\frac{1}{\kappa} A^{-1} C\left(c_{12}, c_{21}\right)^{T}=D_{C}^{-1} C\left(c_{12}, c_{21}\right)^{T},
$$

which equals $\left(c_{12}, c_{21}\right)^{T}$. That is, $\phi_{I}(R)=\left(c_{12}, c_{21}\right)^{T} /\left(c_{12}+c_{21}\right)$, and the result follows. Let us next show that $\phi_{I}$ also satisfies consistency (and a fortiori weak consistency). Let $R=\langle J, a, C\rangle$ be a homogeneous problem and let $\left(v_{i}^{*}\right)_{i \in J}=\phi_{I}(R)$. That is $\left(v_{i}^{*}\right)_{i \in J}$ satisfies

$$
\sum_{j \in J} \frac{c_{i j}}{a_{i}} \frac{a_{j}}{c_{j}} v_{j}^{*}=v_{i}^{*} \text { for } \quad i \in J
$$

Since $c_{j} / a_{j}=\kappa$ for all $i \in J$, pre-multiplying both sides by $c_{i}$ we have

$$
\sum_{j \in J} c_{i j} v_{j}^{*}=v_{i}^{*} c_{i} \text { for all } i \in J .
$$

Now, let $k \in J$ and let $R^{k}=\left\langle J \backslash\{k\},\left(a_{i}\right)_{i \in J \backslash k k\}}, C^{k}\right\rangle$ be the reduced problem with respect to $k$. Note that since $R$ is a homogeneous problem, so is $R^{k}$. Further, $c_{j}^{k}=\sum_{i \in J \backslash k\}} c_{i j}^{k}=\sum_{i \in J} c_{i j}=c_{j}$ for all $j \in J \backslash\{k\}$. By a similar argument, we have that $\phi\left(R^{k}\right)$ solves $\sum_{j \in J} c_{i j}^{k} v_{j}=v_{i} c_{i}$ for $i \in J$. It is enough to show that $\left(v_{i}^{*}\right)_{i \in J \backslash\{k\}}$ satisfies the previous equation. By definition of $c_{i j}^{k}$,

$$
\begin{aligned}
\sum_{j \in J \backslash k k\}} c_{i j}^{k} v_{j}^{*} & =\sum_{j \in J \backslash k\}}\left(c_{i j}+c_{k j} \frac{c_{i k}}{\sum_{t \in J \backslash\{k\}} c_{t k}}\right) v_{j}^{*} \\
& =\sum_{j \in J \backslash k\}} c_{i j} v_{j}^{*}+\sum_{j \in J \backslash k k\}}\left(c_{k j} \frac{c_{i k}}{c_{k}-c_{k k}}\right) v_{j}^{*} .
\end{aligned}
$$

Since by (1), $v_{i}^{*} c_{i}=\sum_{j \in J} c_{i j} v_{j}^{*}$, we have $\sum_{j \in J \backslash k\}\}} c_{i j} v_{j}^{*}=v_{i}^{*} c_{i}-c_{i k} v_{k}^{*}$. Therefore,

$$
\begin{aligned}
\sum_{j \in J \backslash k\}} c_{i j}^{k} v_{j}^{*} & =v_{i}^{*} c_{i}-c_{i k} v_{k}^{*}+\frac{c_{i k}}{c_{k}-c_{k k}} \sum_{j \in J \backslash k k\}} c_{k j} v_{j}^{*} \\
& =v_{i}^{*} c_{i}-c_{i k} v_{k}^{*}+\frac{c_{i k}}{c_{k}-c_{k k}}\left(v_{k}^{*} c_{k}-c_{k k} v_{k}^{*}\right) \\
& =v_{i}^{*} c_{i} .
\end{aligned}
$$


Finally, we shall show that the Invariant method satisfies invariance to splitting of journals. Let $R=\langle J, a, C\rangle$ be a ranking problem and let $R^{\prime}=\left\langle J^{\prime}, a^{\prime}, C^{\prime}\right\rangle$ be a splitting of $R$, where $J^{\prime}=\left\{\left(j, t_{j}\right): j \in J, t_{j} \in T_{j}\right\}, a_{\left(j, t_{j}\right)}^{\prime}=a_{j} / T_{j}$, and $c_{\left(i, t_{i}\right)\left(j, t_{j}\right)}^{\prime}=c_{i j} / T_{i} T_{j}$. Let $\left(v_{i}\right)_{i \in J}=\phi_{I}(R)$ and $\left(v_{\left(i, t_{i}\right)}\right)_{\left(i, t_{i}\right) \in J \times T_{i}}=\phi_{I}\left(R^{\prime}\right)$. By definition of $\phi_{I}(R)$, we know that $v_{i}=\sum_{j \in J}\left(c_{i j} / a_{i}\right)\left(a_{j} / c_{j}\right) v_{j}$, for all $i \in J$. For each $j \in J$, choose $t_{j} \in T_{j}$. We need to show that $v_{\left(i, t_{i}\right)} / v_{\left(j, t_{j}\right)}=v_{i} / v_{j}$ for all $i, j \in J$. It is enough to show that $v_{\left(i, t_{i}\right)}=\sum_{j \in J}\left(c_{i j} / a_{i}\right)\left(a_{j} / c_{j}\right) v_{\left(j, t_{j}\right)}$ for all $i \in J$. By definition of $\phi_{I}\left(R^{\prime}\right)$, we have

$$
\begin{aligned}
v_{\left(i, t_{i}\right)} & =\sum_{j \in J} \sum_{t_{j} \in T_{j}} \frac{c_{\left(i, t_{i}\right)\left(j, t_{j}\right)}^{\prime}}{a_{\left(i, t_{i}\right)}^{\prime}} \frac{a_{\left(j, t_{j}\right)}^{\prime}}{c_{\left(j, t_{j}\right)}^{\prime}} v_{\left(j, t_{j}\right)} \\
& =\sum_{j \in J} \sum_{t_{j} \in T_{j}} \frac{c_{i j} / T_{i} T_{j}}{a_{i} / T_{i}} \frac{a_{j} / T_{j}}{c_{j} / T_{j}} v_{\left(j, t_{j}\right)} \\
& =\sum_{j \in J} \frac{c_{i j}}{a_{i}} \frac{a_{j}}{c_{j}} v_{\left(j, t_{j}\right)},
\end{aligned}
$$

which is what we wanted to prove.

Next we shall show that a ranking method that satisfies the four axioms must be the Invariant method.

LEMMA: Let $\phi: \mathcal{R} \rightarrow \Delta$ be a ranking method that satisfies weak homogeneity, invariance to reference intensity, and weak consistency. Then, $\phi$ coincides with the Invariant method for all isoarticle problems.

PROOF: The proof is by induction on the number of journals. Let $R=\langle\{i, j\}, a, C\rangle$ be a twojournal ranking problem such that $a_{i}=a_{j}$. We need to show that $\phi(R)=\left(c_{i j} /\left(c_{i j}+c_{j i}\right), c_{j i} /\left(c_{i j}+\right.\right.$ $\left.\left.c_{j i}\right)\right) \equiv \phi_{I}(R)$. By invariance to reference intensity, we can assume that the entries of each column of $C$ add up to one, and by weak homogeneity, $\phi_{i}(R) / \phi_{j}(R)=c_{i j} / c_{j i}$. Assume now that $\phi$ coincides with the Invariant method for all $n$-journal problems in which all journals have the same number of articles, and let $R=\langle J, a, C\rangle$ be an $n+1$ journal problem in that class. Since both $\phi$ and $\phi_{I}$ satisfy invariance to reference intensity, we can assume without loss of generality that the entries of each column of $C$ add up to one. By the induction hypothesis, for all $k \in J$ we have $\phi\left(R^{k}\right)=\phi_{I}\left(R^{k}\right)$. But then, by weak consistency of both $\phi$ and $\phi_{I}$, for all $k \in J$

$$
\frac{\phi_{i}(R)}{\phi_{j}(R)}=\frac{\phi_{i}\left(R^{k}\right)}{\phi_{j}\left(R^{k}\right)}=\frac{\left(\phi_{I}\right)_{i}\left(R^{k}\right)}{\left(\phi_{I}\right)_{j}\left(R^{k}\right)}=\frac{\left(\phi_{I}\right)_{i}(R)}{\left(\phi_{I}\right)_{j}(R)} \quad \text { for all } i, j \in J .
$$

This implies that $\phi(R)=\phi_{I}(R)$.

Q.E.D.

In order to complete the proof, we need to show that if $\phi$ satisfies all the axioms and coincides with the Invariant method for all isoarticle ranking problems, then $\phi$ is, in fact, the Invariant method. Let $R=\left\langle J,\left(a_{j}\right)_{j \in J},\left(c_{i j}\right)_{(i, j) \in J \times J}\right\rangle$ be a ranking problem and let $A=\operatorname{diag}\left(a_{j}\right)_{j \in J}$. Let $\phi$ be a ranking method that satisfies all the foregoing axioms. We need to show that $\phi(R)$ solves the equation $v=A^{-1} C D_{C}^{-1} A v$. Define $v^{*} \in \Delta_{J}$ to be the only solution to $v=C D_{C}^{-1} v$. That is, $v^{*}$ would be the vector of relative valuations awarded by the Invariant method if all the journals in $J$ had the same number of articles. Therefore, $A^{-1} v^{*}$ solves $v=A^{-1} C D_{C}^{-1} A v$. Then, we need to show that $\phi(R)=A^{-1} v^{*} /\left\|A^{-1} v^{*}\right\|$. Let $G$ be the greatest common divisor of $\left(a_{j}\right)_{j \in J}$ and let $T_{j}=a_{j} / G$. We will split each journal $j \in J$ into $T_{j}$ identical journals. The set of journals will be $J^{\prime}=\left\{\left(j, t_{j}\right): j \in J, t_{j} \in T_{j}\right\}$. The number of articles of journal $\left(j, t_{j}\right)$, for $j \in J, t_{j} \in T_{j}$, is given by $a_{\left(j, t_{j}\right)}=a_{j} / T_{j}=G$. The new matrix of citations is $C^{\prime}=\left(c_{\left(i, t_{i}\right)\left(j, t_{j}\right)}^{\prime}\right)$, where $c_{\left(i, t_{i}\right)\left(j, t_{j}\right)}^{\prime}=c_{i j} /\left(T_{i} T_{j}\right)$. Summarizing, $R^{\prime}=\left\langle J^{\prime},\left(a_{\left(j, t_{j}\right)}\right)_{j \in J, t_{j} \in T_{j}},\left(c_{\left(i, t_{i}\right)\left(j, t_{j}\right)}^{\prime}\right)_{\left(\left(i, t_{i}\right),\left(j, t_{j}\right)\right) \in J^{\prime} \times J^{\prime}}\right\rangle$. Since $R^{\prime}$ is a ranking problem where all journals have the same number of articles, we know by the previous lemma that $\phi\left(R^{\prime}\right)$ is 
the solution to $C^{\prime} D_{C}^{\prime-1} v=v$, where $C^{\prime} D_{C}^{\prime-1}$ is the normalized matrix of $C^{\prime}$. Denote this unique solution by $\bar{v}$. Note that $\bar{v}$ is a $\left|J^{\prime}\right|$-dimensional vector. However, by the above lemma, $\bar{v}_{\left(i, t_{i}\right)}=\bar{v}_{\left(i, s_{i}\right)}$ for all $i \in J$ and for all $t_{i}, s_{i} \in T_{i}$, since the Invariant method assigns equal values to identical journals. Denote this common value by $\bar{v}_{i}, i \in J$. We shall show that, for all $i \in J, T_{i} \bar{v}_{i}=\sum_{j \in J} T_{j} \bar{v}_{j} c_{i j} / c_{j}$. To see this, note that

$$
\begin{aligned}
T_{i} \bar{v}_{i} & =T_{i} \bar{v}_{\left(i, t_{i}\right)} \\
& =T_{i} \sum_{j \in J} \sum_{t_{j} \in T_{j}} \bar{v}_{\left(j, t_{j}\right)} c_{\left(i, t_{i}\right)\left(j, t_{j}\right)}^{\prime} / c_{\left(j, t_{j}\right)}^{\prime} \\
& =T_{i} \sum_{j \in J} \sum_{t_{j} \in T_{j}} \bar{v}_{j} \frac{c_{i j}}{T_{i} T_{j}} T_{j} / c_{j} \\
& =\sum_{j \in J} \sum_{t_{j} \in T_{j}} \bar{v}_{j} c_{i j} / c_{j} \\
& =\sum_{j \in J} \bar{v}_{j} T_{j} c_{j i} / c_{j} .
\end{aligned}
$$

Therefore, $\bar{v}_{i} T_{i}=v_{i}^{*}$. Dividing both sides by $a_{i}$ we get $\bar{v}_{i} / G=v_{i}^{*} / a_{i}$ for all $i \in J$. This means that the vectors $A^{-1} v^{*}$ and $\left(\bar{v}_{i}\right)_{i \in J}$ are proportional. But by the invariance of $f$ to splitting of journals, we know that the vectors $\phi(R)$ and $\left(\bar{v}_{i}\right)_{i \in J}$ are proportional too, which implies that $A^{-1} v^{*}$ and $\phi(R)$ are proportional. Since $\|\phi(R)\|=1$, we must have $\phi(R)=A^{-1} v^{*} /\left\|A^{-1} v^{*}\right\|$.

\section{APPENDIX B: INDEPENDENCE OF THE AXIOMS}

In order to see that the weak homogeneity axiom is not implied by the other three axioms, consider the method $\phi_{E}: \mathcal{R} \rightarrow \Delta$ defined by $\phi_{E}(J, a, C)=(1 /|J|, \ldots, 1 /|J|)^{T}$. It is easy to check that $\phi_{E}$ satisfies invariance to reference intensity, consistency, and invariance to splitting of journals, but it does not satisfy weak homogeneity.

We will now build a ranking method that satisfies all axioms except for weak consistency. Let $R=\langle J, a, C\rangle$ be a ranking problem. We say that journal $i \in J$ is similar to journal $j \in J$ if $c_{i k} / c_{i s}=c_{j k} / c_{j s}$ and $c_{k i} / c_{s i}=c_{k j} / c_{s j}$ for all $k, s \in J$. The similarity relation is an equivalence relation. Therefore, it partitions the set of journals into equivalence classes. Denote by [i] the equivalence class that contains $i \in J$ and consider a ranking problem $R^{2}=\left\langle J^{2}, a^{2}, C^{2}\right\rangle$, where $J^{2}$ is the set of equivalence classes of $J$ induced by the above similarity relation, ${ }^{6} a_{[i]}^{2}=\sum_{t_{i} \in[i]} a_{t_{i}}$, for $[i] \in J^{2}$ and $c_{[i][j]}=\sum_{t_{i} \in[i]} \sum_{t_{j} \in[j]} c_{t_{i} t_{j}}$. That is, the ranking problem $R^{2}$ is obtained from $R$ by merging all the journals that are similar to each other. Repeat the process starting from $R^{2}$ until the limiting ranking problem $R^{\infty}$ has no two journals that are similar to each other. Consider the ranking method $\phi_{S C}: \mathcal{R} \rightarrow \Delta$, defined as follows. Given a ranking problem $R=\langle J, a, C\rangle$ let $\tilde{R}=\left\langle J, a, C D_{C}^{-1}\right\rangle$ be the associated normalized problem and let $\tilde{R}^{\infty}$ be the problem that results from the above merging of similar journals, applied to the normalized problem. The method $\phi_{S C}$ assigns to each journal $i \in J$ a value $v_{i}=\lambda v_{[i]}^{*}$, where $v_{[i]}^{*}$ is the value awarded to the equivalence class that contains $i \in J$ by the Modified Counting method when applied to the problem $\tilde{R}^{\infty}$ and $\lambda$ is chosen so that $\left(v_{i}\right)_{i \in J} \in \Delta_{J}$. This method satisfies invariance to reference intensity since it applies the Modified Counting method to the normalized problem. It can be checked that it satisfies weak homogeneity. It also satisfies invariance to splitting of journals, because by splitting a journal one gets similar subjournals. This method, however, does not satisfy weak consistency.

${ }^{6}$ To be accurate, $J^{2} \subseteq J$ contains exactly one element of each equivalence class. 
To see that invariance to reference intensity is not implied by the other axioms, consider the Liebowitz-Palmer (LP) method. This method satisfies homogeneity because for any matrix of the form

$$
A^{-1} C=\left(\begin{array}{cc}
\kappa / c_{1} & 0 \\
0 & \kappa / c_{2}
\end{array}\right)\left(\begin{array}{cc}
c_{1}-c_{21} & c_{12} \\
c_{21} & c_{2}-c_{12}
\end{array}\right)
$$

we have $A^{-1} C\left(c_{12}, c_{21}\right)^{T}=\kappa\left(c_{12}, c_{21}\right)^{T}$. To see that it also satisfies weak consistency, let $\left(v_{i}^{*}\right)_{i \in J}=$ $\phi_{L P}(R)$. That is $\left(v_{i}^{*}\right)_{i \in J} \in \Delta_{J}$ solves $v=\lambda A^{-1} C v$, for some $\lambda \neq 0$. Since $D_{C} A^{-1}=\kappa I$, we have that $D_{C} v^{*}=\lambda \kappa C v^{*}$. The rest of the proof is analogous to the proof that the Invariant method satisfies consistency. The proof that the LP method satisfies invariance to splitting of journals is similar to the analogous proof for the Invariant method, and is left to the reader. Finally, the LP method does not satisfy Invariance to reference intensity.

Lastly, consider the ranking method $\phi: \mathcal{R} \rightarrow \Delta$, where $\phi(J, a, C)$ is the only eigenvector in $\Delta_{J}$ of the matrix $\left(A^{2}\right)^{-1} C D_{C}^{-1} A^{2}$. It satisfies invariance to reference intensity, weak homogeneity, and weak consistency. It does not satisfy invariance to splitting of journals.

\section{REFERENCES}

AMIR, R. (2002): "Impact-Adjusted Citations as a Measure of Journal Quality," CORE Discussion Paper 2002/74, Université Catholique de Louvain.

Bush, W. C., P. W. HAMElman, AND R. J. StaAF (1974): "A Quality Index for Economic Journals," Review of Economics and Statistics, 56, 123-125.

Ellison, G. (2002): "The Slowdown of the Economics Publishing Process," Journal of Political Economy, 110, 947-993.

GARFIELD, E. (1972): "Citation Analysis as a Tool in Journal Evaluation,” Science, 178, 471-479.

HAMERMESH, D. S., G. E. Johnson, AND B. A. Weisbrod (1982): "Scholarship, Citations and Salaries: Economic Rewards in Economics," Southern Economic Journal, 49, 472-481.

LABAND, D. N., AND M. J. PIETTE (1994a): "Favoritism Versus Search for Good Papers: Empirical Evidence Regarding the Behavior of Journal Editors," Journal of Political Economy, 102, 194-203.

_ (1994b): “The Relative Impacts of Economics Journals: 1970-1990,” Journal of Economic Literature, 32, 640-666.

LABAND, D. N., AND R. D. TOLLISON (2000): “Intellectual Collaboration,” Journal of Political Economy, 108, 632-662.

Liebowitz, S., AND J. PALMER (1984): “Assessing the Relative Impacts of Economic Journals," Journal of Economic Literature, 22, 77-88.

Minc, H. (1988): Nonnegative Matrices. New York: John Wiley \& Sons.

Page, L., S. BRIN, R. Motwani, AND T. Winograd (1998): “The PageRank Citation Ranking: Bringing Order to the Web,” Technical Report, Stanford University.

PINSKI, G., AND F. NARIN (1976): "Citation Influence for Journal Aggregates of Scientific Publications: Theory, with Applications to the Literature of Physics," Information Processing and Management, 12, 297-312.

Posner, R. A. (2000): "An Economic Analysis of the Use of Citations in the Law," American Law and Economics Review, 2, 381-406.

SAUER, R. D. (1988): "Estimates of the Returns to Quality and Coauthorship in Economic Academia," Journal of Political Economy, 96, 855-866.

STIGLER, G. J., S. M. STIGLER, AND C. FRIEDLAND (1995): “The Journals of Economics,” Journal of Political Economy, 103, 331-359.

TUCKMAN, H. P., AND J. LEAHEY (1975): “What Is an Article Worth?” Journal of Political Economy, 88, 951-967. 